Springer-Verlag.

\title{
Limits of Task-based Parallelism in Irregular Applications
}

\author{
Barbara Kreaseck Dean Tullsen Brad Calder \\ Department of Computer Science and Engineering \\ University of California, San Diego \\ La Jolla, CA 92093-0114 \\ \{kreaseck, tullsen, calder\}@cs.ucsd.edu
}

\begin{abstract}
Traditional parallel compilers do not effectively parallelize irregular applications because they contain little loop-level parallelism. We explore Speculative Task Parallelism (STP), where tasks are full procedures and entire natural loops. Through profiling and compiler analysis, we find tasks that are speculatively memory- and control-independent of their neighboring code. Via speculative futures, these tasks may be executed in parallel with preceding code when there is a high probability of independence. We estimate the amount of STP in irregular applications by measuring the number of memoryindependent instructions these tasks expose. We find that 7 to $22 \%$ of dynamic instructions are within memory-independent tasks, depending on assumptions.
\end{abstract}

\section{Introduction}

Today's microprocessors rely heavily on instruction-level parallelism (ILP) to gain higher performance. Flow control imposes a limit to available ILP in single-threaded applications [8]. One way to overcome this limit is to find parallel tasks and employ multiple flows of control (threads). Task-level parallelism (TLP) arises when a task is independent of its neighboring code. We focus on finding these independent tasks and exploring the resulting performance gains.

Traditional parallel compilers exploit one variety of TLP, loop level parallelism (LLP), where loop iterations are executed in parallel. LLP can overwhelming be found in numeric, typically FORTRAN programs with regular patterns of data accesses. In contrast, general purpose integer applications, which account for the majority of codes currently run on microprocessors, exhibit little LLP as they tend to access data in irregular patterns through pointers. Without pointer disambiguation to analyze data access dependences, traditional parallel compilers cannot parallelize these irregular applications and ensure correct execution.

In this paper we explore task-level parallelism in irregular applications by focusing on Speculative Task Parallelism (STP), where tasks are speculatively executed in parallel under the following assumptions: 1) tasks are full procedures or entire natural loops, 2) tasks are speculatively memory-independent and controlindependent, and 3) our architecture allows the parallelization of tasks via speculative futures (discussed below). Figure 1 illustrates STP, showing a dynamic instruction stream where a task Y has no memory access conflicts with a group of instructions, $\mathrm{X}$, that precede $\mathrm{Y}$. The shorter of $\mathrm{X}$ and $\mathrm{Y}$ determines the overlap of memory-independent instructions as seen in Figures 1(b) and 1(c). In the absence of any register dependences, $\mathrm{X}$ and $\mathrm{Y}$ may be executed in parallel, resulting in shorter execution time. It is hard for traditional parallel compilers of pointer-based languages to expose this parallelism. 


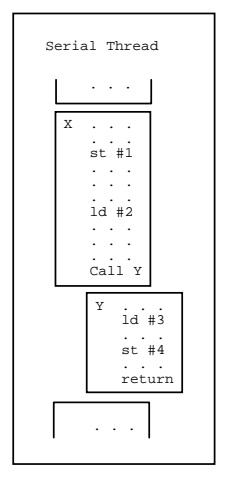

(a)

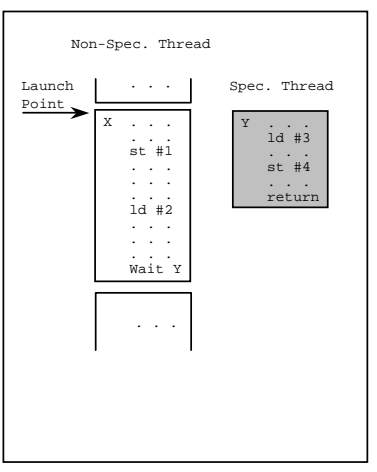

(b)

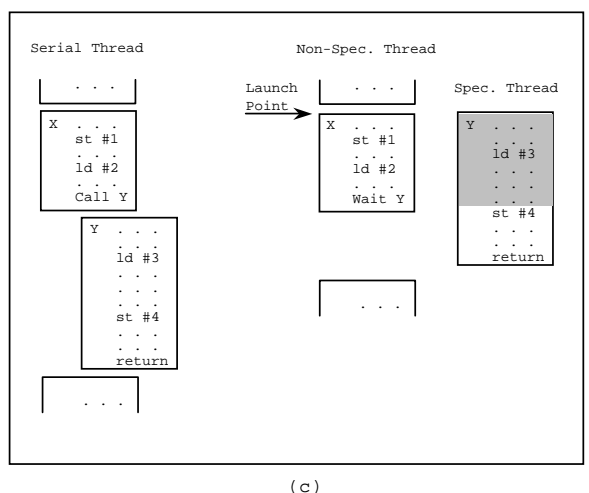

Figure 1: STP example: (a) shows a section of code where the task $Y$ is known to be memory-independent of the preceding code $X$. $(b)$ the shaded region shows memory- and control-independent instructions that are essentially removed from the critical path when $Y$ is executed in parallel with $X .(c)$ when task $Y$ is longer than $X$.

The goals of this paper are to identify such regions within irregular applications and to find the number of instructions that may thus be removed from the critical path. This number represents the maximum possible STP. To facilitate our discussion, we offer the following definitions.

A task, exemplified by $\mathrm{Y}$ in Figure 1, is a bounded set of instructions inherent to the application. Two sections of code are memory-independent when neither contains a store to a memory location that the other accesses. When all load/store combinations of the type [load,store], [store,load] and [store,store] between two tasks, $\mathrm{X}$ and $\mathrm{Y}$, access different memory locations, $\mathrm{X}$ and $\mathrm{Y}$ are said to be memory-independent. A launch point is the point in the code preceding a task where the task may be initiated in parallel with the preceding code. This point is determined through profiling and compiler analysis. A launched task is one that begins execution from an associated launch point on a different thread.

Because the biggest barrier to detecting independence in irregular codes is memory disambiguation, we identify memory-independent tasks using a profile-based approach and measure the amount of STP by estimating the amount of memory-independent instructions those tasks expose. As successive executions may differ from the profiled execution, any launched task would be inherently speculative. One way of launching a task in parallel with its preceding code is through a parallel language construct called a future. A future conceptually forks a thread to execute the task and identifies an area of memory in which to relay status and results. When the original thread needs the results of the futured task, it either waits on the futured task thread, or in the case that the task was never futured due to no idle threads, it executes the futured task itself.

To exploit STP, we assume a speculative machine that supports speculative futures. Such a processor could speculatively execute code in parallel when there is a high probability of independence, but no guarantee. Our work identifies launch points for this speculative machine, and estimates the parallelism available to such a machine. With varying levels of control and memory speculation, 7 to $22 \%$ of dynamic instructions are within tasks that are found to be memory-independent, on a set of irregular applications for which traditional methods of parallelization are ineffective.

In the next section we discuss related work. Section 3 contains a description of how we identify and quantify STP. Section 4 describes our experiment methodology and Section 5 continues with some results. Implementation issues are highlighted in Section 6, followed by a summary in Section 7. 


\section{Related Work}

In order to exploit Speculative Task Parallelism, a system would minimally need to include multiple flows of control and memory disambiguation to aid in mis-speculation detection. Current proposed structures that aid in dynamic memory disambiguation are implemented in hardware alone [3] or rely upon a compiler [5, 4]. All minimally allow loads to be speculatively executed above stores and detect write-after-read violations that may result from such speculation.

Some multithreaded machines [21, 19, 2] and single-chip multiprocessors [6, 7] facilitate multiple flows of control from a single program, where flows are generated by compiler and/or dynamically. All of these architectures could exploit non-speculative TLP if the compiler exposed it, but only Hydra [6] could support STP without alteration.

Our paper examines speculatively parallel tasks in non-traditionally parallel applications. Other proposed systems, displaying a variety of characteristics, also use speculation to increase parallelism. They include Multiscalar processors [16, 12, 20], Block Structured Architecture [9], Speculative Thread-level Parallelism [15, 14], Thread-level Data Speculation [18], Dynamic Multithreading Processor [1], and Data Speculative Multithreaded hardware architecture $[11,10]$.

In these systems, the type of speculative tasks include fixed-size blocks [9], one or more basic blocks [16], dynamic instruction sequences [18], loop iterations [15, 11], instructions following a loop [1], or following a procedure call $[1,14]$. These tasks were identified dynamically at run-time $[11,1]$, statically by compilers [20, 9, 14], or by hand [18]. The underlying architectures include traditional multiprocessors [15, 18], non-traditional multiprocessors $[16,9,10]$, and multithreaded processors $[1,11]$.

Memory disambiguation and mis-speculation detection was handled by an Address Resolution Buffer [16], the Time Warp mechanism of time stamping requests to memory [9], extended cache coherence schemes [14, 18], fully associative queues [1], and iteration tables [11]. Control mis-speculation was always handled by squashing the mis-speculated task and any of its dependents. While a few handled data mis-speculations by squashing, one rolls back speculative execution to the wrong data speculation [14] and others allow selective, dependent re-execution of the wrong data speculation $[9,1]$.

Most systems facilitate data flow by forwarding values produced by one thread to any consuming threads $[16,9,18,1,11]$. A few avoid data mis-speculation through synchronization $[12,14]$. Some systems enable speculation by value prediction using last-value $[1,14,11]$ and stride-value predictors $[14,11]$.

STP identifies a source of parallelism that is complimentary to that found by most of the systems above. Armed with a speculative future mechanism, these systems may benefit from exploiting STP.

\section{Finding Task-based Parallelism}

We find Speculative Task Parallelism by identifying all tasks that are memory-independent of the code that precedes the task. This is done through profiling and compiler analysis, collecting data from memory access conflicts and control flow information. These conflicts determine proposed launch points that mark the memory dependences of a task. Then for each task, we traverse the control flow graph (CFG) in reverse control flow order to determine launch points based upon memory and control dependences. Finally, we estimate the parallelism expected from launching the tasks early. The following explain the details of our approach to finding STP.

\section{Task Selection}

The type of task chosen for speculative execution directly affects the amount of speculative parallelism found in an application. Oplinger, et. al. [14], found that loop iterations alone were insufficient to make speculative thread-level parallelism effective for most programs. To find STP, we look at three types of 


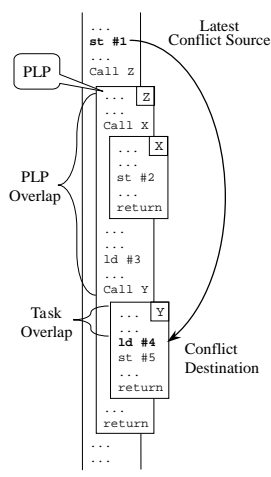

(a)

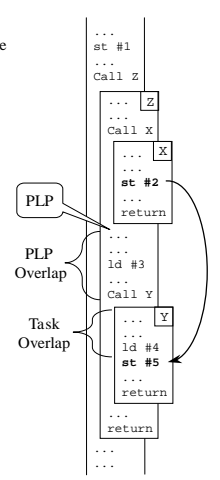

(b)

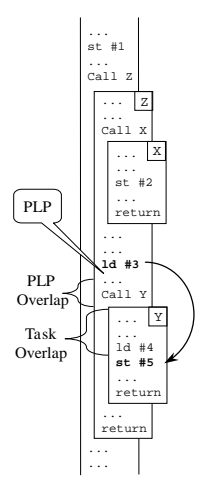

(c)

Figure 2: PLP Locations: conflict source, conflict destination, PLP candidate, PLP overlap, and task overlap, when the latest conflict source is (a) before the calling routine, $(b)$ within a sibling task $(c)$ within the calling routine.

tasks: leaf procedures (procedures that do not call any other procedure), non-leaf procedures, and entire natural loops. When profiling a combination of task types, we profile them concurrently, exposing memoryindependent instructions within an environment of interacting tasks.

Although all tasks of the chosen type(s) are profiled, only those that expose at least a minimum number of memory-independent instructions are chosen to be launched early. The final task selection is made after evaluating memory and control dependences to determine actual launch points.

\section{Memory Access Conflicts}

Memory access conflicts are used to determine the memory dependences of a task. They occur when two load/store instructions access the same memory region. Only a subset of memory conflicts that occur during execution are useful for calculating launch points. Useful conflicts span task boundaries and are of the form [load, store], [store, load], or [store, store]. We also disregard stores or loads due to register saves and restores across procedure calls. We call the conflicting instruction preceding the task the conflict source, and the conflicting instruction within the task is called the conflict destination. Specifically, when the conflict destination is a load, the conflict source will be the last store to that memory region that occurred outside the task. When the conflict destination is a store, the conflict source will be the last load or store to that memory region that occurred outside the task.

\section{Proposed Launch Points}

The memory dependences for a task are marked, via profiling, as proposed launch points (PLPs). A PLP represents the memory access conflict with the latest (closest) conflict source in the dynamic code preceding one execution of that task. Exactly one PLP is found for each dynamic task execution. In our approach, launch points for a task occur only within the task's calling region, limiting the amount and scope of executable changes that would be needed to exploit STP. Thus, PLPs must also lie within a task's calling routine.

Figure 2 contains an example that demonstrates the latest conflict sources and their associated PLPs. Task $\mathrm{Z}$ calls tasks $\mathrm{X}$ and $\mathrm{Y}$. $\mathrm{Y}$ is the currently executing task in the example and $\mathrm{Z}$ is its calling routine. When the conflict source occurs before the beginning of the calling routine, as in Figure 2(a), the PLP is directly before the first instruction of the task $\mathrm{Z}$. When the conflict source occurs within a sibling task or its child tasks, as in Figure 2(b), the PLP immediately follows the call to the sibling task. In Figure 2(c), the conflict source is a calling routine instruction and the PLP immediately follows the conflict source. 

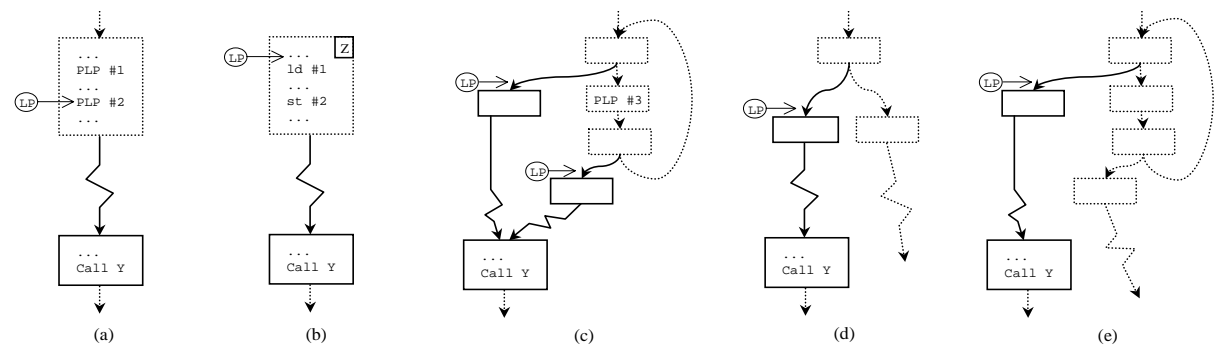

Figure 3: Task Launch Points: Dotted areas have not been fully visited by the back-trace for task Y. (a) CFG block contains a PLP. (b) CFG block is calling routine head. (c) Loop contains a PLP. (d) Incompletely visited CFG block. (e) Incompletely visited loop.

Two measures of memory-independence are associated with each PLP. They are the PLP overlap and the task overlap, as seen in Figure 2. The PLP overlap represents the number of dynamic instructions found between the PLP and the beginning of the task. The task overlap represents the number of dynamic instructions between the beginning of the task and the conflict destination. With PLPs determined by memory dependences that are dynamically closest to the task call site, and by recording the smallest task overlap, we only consider conservative, safe locations with respect to the profiling dataset.

\section{Task Launch Points}

Both memory dependences and control dependences influence the placement of task launch points. Our initial approach to exposing STP determines task launch points that provide two guarantees. First, static control dependence is preserved: all paths from a task launch point lead to the original task call site. Second, profiled memory dependence is preserved: should the threaded program be executed on the profiling dataset, all instructions between the task launch point and the originally scheduled task call site will be free of memory conflicts. Variations which relax these guarantees are described in Section 5.2.

For each task, we recursively traverse the CFG in reverse control flow order starting from the original call site, navigating conditionals and loops, to identify task launch points. We use two auxiliary structures: a stalled block list to hold incompletely visited blocks, and a stalled loop list to hold incompletely visited loops. There are five conditions under which we record a task launch point. These conditions are described below. The first three will halt recursive back-tracing along the current path. As illustrated in Figure 3, we record task launch points:

a. when the current CFG block contains a PLP for that task. The task launch point is the last PLP in the block.

b. when the current CFG block is the head of the task's calling routine and contains no PLPs. The task launch point is the first instruction in the block.

c. when the current loop contains a PLP for that task. Back-tracing will only get to this point when it visits a loop, and all loop exit edges have been visited. As this loop is really a sibling of the current task, task launch points are recorded at the end of all loop exit edges.

d. for blocks that remain on the stalled block list after all recursive back-tracing has exited. A task launch point is recorded only at the end of each visited successor edge of the stalled block.

e. for loops that remain on the stalled loop list after all recursive back-tracing has exited. A task launch point is recorded only at the end of each visited loop exit edge.

Each task launch point indicates a position in the executable in which to place a task future. At each task's original call site, a check on the status of the future will indicate whether to execute the task serially or wait on the result of the future. 


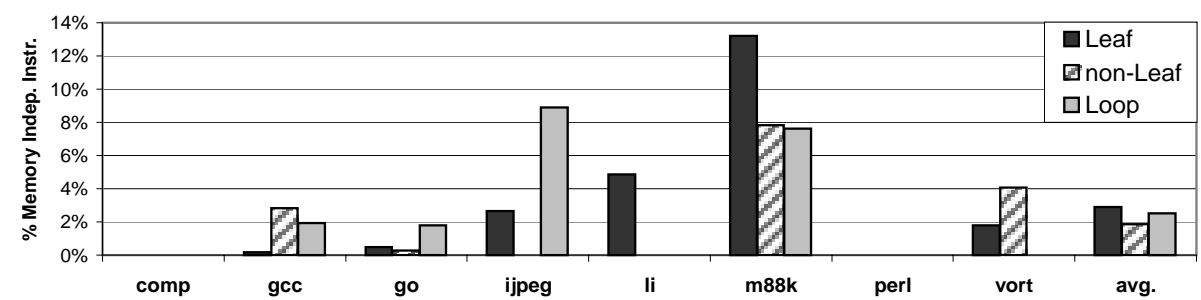

Figure 4: Task Types: Individual data points identify memory independent instructions as a percentage of all instructions profiled and represent our starting configuration.

\section{Parallelization Estimation}

We estimate the number of memory-independent instructions that would have been exposed had the tasks been executed at their launch points during the profile run. Our approach ensures that each instruction is counted as memory-independent at most once. When the potential for instruction overlap exceeds the task selection threshold the task is marked for STP. We use the total number of claimed memory-independent instructions as an estimate of the limit of STP available on our hypothetical speculative machine.

\section{Methodology}

To investigate STP, we used the ATOM profiling tools [17] and identified natural loops as defined by Muchnick [13]. We profiled the SPECint95 suite of benchmark programs. Each benchmark was profiled for 650 million instructions. We used the reference datasets on all benchmarks except compress. For compress, we used a smaller dataset, in order to profile a more interesting portion of the application.

We measure STP by the number of memory-independent task instructions that would overlap preceding non-task instructions should a selected task be launched (as a percentage of all dynamic instructions).

The task selection threshold comprises two values, both of which must be exceeded. For all runs, the task selection threshold was set at 25 memory-independent instructions per task execution and a total of $0.2 \%$ of instructions executed. We impose this threshold to compensate for the expected overhead of managing speculative threads and to enable allocation of limited resources to tasks exposing more STP.

Our results show a limit to STP exposed by the launched execution of memory-independent tasks. No changes, such as code motion, were made or assumed to have been made to the original benchmark codes that would heighten the amount of memory-independent instructions. Overhead due to thread creation, along with wakeup and commit, will be implementation dependent, and thus is not accounted for. Register dependences between the preceding code and the launched task were ignored. Therefore, we show an upper bound to the amount of STP in irregular applications.

\section{Results}

We investigated the amount of Speculative Task Parallelism under a variety of assumptions about task types, memory conflict granularity, control and memory dependences. Our starting configuration includes profiling at the page-level (that is, conflicts are memory accesses to the same page) with no explicit speculation and is thus our most conservative measurement.

\subsection{Task Type}

We first look to see which task types exhibit the most STP. We then explore various explicit speculation opportunities to find additional sources of STP. Finally, we investigate any additional parallelism that might 

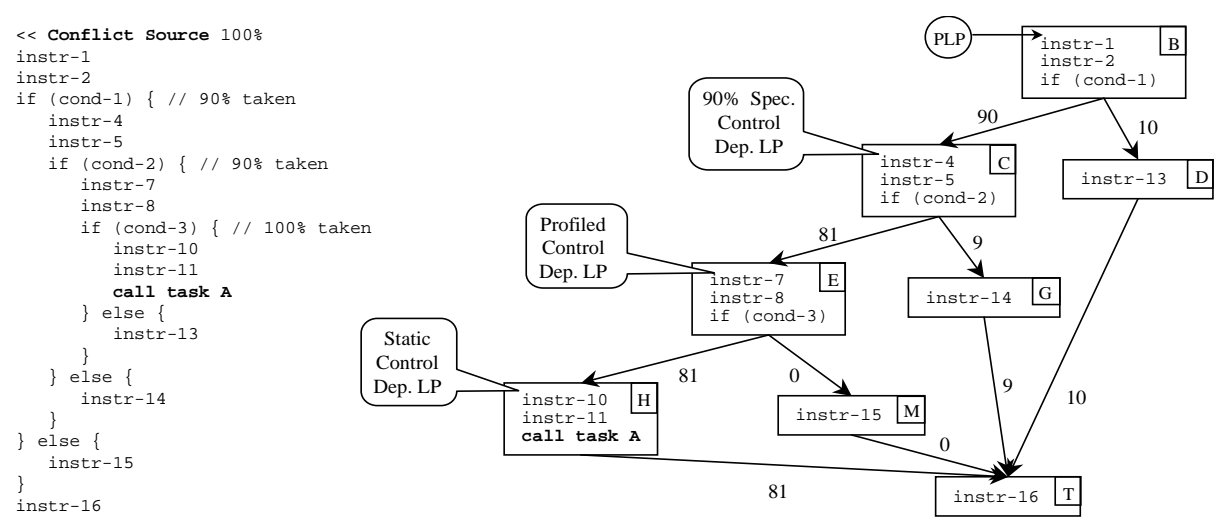

Figure 5: Control Dependence Speculation: Using the profile information that the conditions are true 90\% , 90\%, and 100\%, respectively, profiled control dependence and speculative control dependence allow task A to be launched outside of the inner if statement. The corresponding CFG displays the launch points as placed by each type of control dependence. The edge frequencies reflect that the code was executed 100 times.

be exposed by profiling at a finer memory granularity.

We investigate leaf procedures, non-leaf procedures, and entire natural loops. We profiled these three task types to see if any one task type exhibited more STP than the others. Figure 4 shows that, on average, a total of $7.3 \%$ of the profiled instructions were identified as memory independent, with task type contributions differing by less than $1 \%$ of the profiled instructions. This strongly suggests that all task types should be considered for exploiting STP. The succeeding experiments include all three task types in their profiles.

\subsection{Explicit Speculation}

The starting configuration places launch points conservatively, with no explicit control or memory dependence speculation. Because launched tasks will be implicitly speculative when executed with different datasets, our hypothetical machine must already support speculation and recovery. We explore the level of STP exhibited by explicit speculation, first, by speculating on control dependence, where the launched task may not actually be needed. Next, we speculate on memory dependence, where the launched task may not always be memory-independent of the preceding code. Finally, we speculate on both memory and control dependences by exploiting the memory-independence of the instructions within the task overlap.

Our starting configuration determines task launch points that preserve static control dependences, such that all paths from the task's launch points lead to the original task call site. Thus, a task that is statically control dependent upon a condition whose outcome is constant, or almost constant, throughout the profile, will not be selected, even though launching this task would lead to almost no mis-speculations. We considered two additional control dependence schemes that would be able to exploit the memory-independence of this task.

Profiled control dependences exclude any static control dependences that are based upon branch paths that are never traversed. When task launch points preserve profiled control dependences, all traversed paths from the launch points lead to the original call site.

When task launch points preserve speculative control dependences, all frequently traversed paths from the launch points lead to the original call site. The amount of speculation is controlled by setting a minimum frequency percentage, $c$. For example, when $c$ is set to 90 , then at least $90 \%$ of the traversed paths from the launch points must lead to the original call site.

In Figure 5, the call statement of task A is statically control dependent on all three if-statements. The 


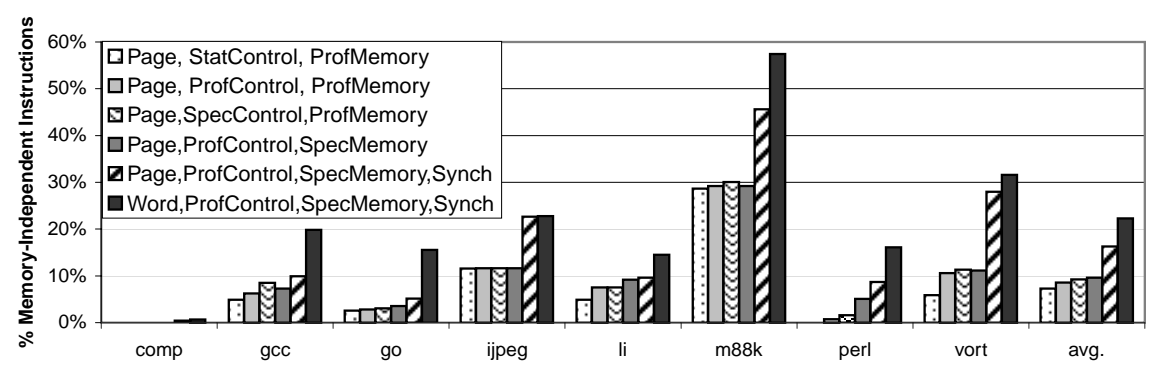

Figure 6: Memory-independent instructions reported as a percentage of all instructions profiled. Page = Page-level profiling, Word $=$ Word-level profiling, ProfControl $=$ Profiled control dependence, SpecControl = Speculative control dependence, SpecMemory = Speculative memory dependence, Synch = Early start with synchronization.

corresponding CFG in Figure 5 highlights the launch points as determined by the three control dependence options. All paths beginning with block $\mathrm{H}$, all traversed paths beginning with block $\mathrm{E}$, and $90 \%$ of the traversed paths beginning with block $\mathrm{C}$ lead to the call of task $\mathrm{A}$. Therefore, the static control dependence launch point is before block $\mathrm{H}$, the profiled control dependence launch point is before block $\mathrm{E}$, and with $c$ set to 90 , the speculative control dependence launch point is before block $\mathrm{C}$.

The price of using speculative control dependences will be the waste of resources used to speculatively initiate a launched task when the executed path does not lead to the task call site. These extra launches can be squashed at the first mis-speculated conditional.

The first three bars per benchmark in Figure 6 show the effect of control dependence speculation. The bars display static control dependence, profiled control dependence, and speculative control dependence at $c=90$, respectively. On average, profiled control dependence exposed an additional $1.3 \%$ of dynamic instructions as memory-independent, while speculative control dependence only exposed an additional $0.6 \%$ over profiled.

The choice of using profiled or speculative control dependence will be influenced by the underlying architecture, and the degree to which speculative threads compete with non-speculative for resources. Further results in this paper use profiled control dependence, due to the low gain from speculative control dependence.

Memory dependence provides another opportunity for explicit speculation. Our starting configuration determines launch points that preserve profiled memory dependences such that all instructions between each launch point and its original task call site are memory-independent of the task. This approach results in a conservative, but still speculative, placement of launch points.

We also consider the less conservative approach of determining task launch points by speculative memory dependences, which ignores profiled memory conflicts that occur infrequently. The amount of speculation is controlled by setting a minimum frequency percentage, $m$. For example, when $m$ is set to 90 , then at least $90 \%$ of the traversed paths from the task launch points to the original call site must be memoryindependent of the task. Using speculative memory dependences is especially attractive when PLPs are far apart, and the ones nearest the task call site seldom cause a memory conflict.

We examine the effect of task launch points that preserve speculative memory dependence at $m=90$ (the fourth bar in Figure 6). Speculative memory dependence provides small increases in parallelism. Despite the small gains, we include task launch points determined by speculative memory dependence for the remaining results.

By placing launch points (futures) at control dependences or memory dependences (PLPs), we have used the limited synchronization inherent within futures to synchronize these dependences with the beginning 


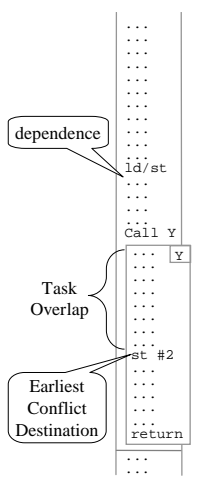

(a)

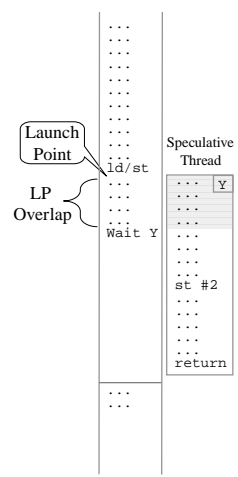

(b)

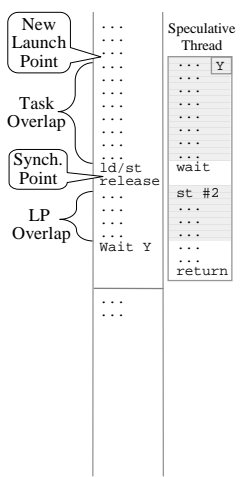

(c)

Figure 7: Synchronization Points: Gray areas represent memory-independent instructions. (a) a task with a large amount of task overlap on a serial thread. (b) When the dependence is used as a launch point, only LP overlap contributes to memory-independence. (c) By synchronizing the dependence with the earliest conflict destination, both LP overlap and task overlap contribute to memory-independence.

of the speculative task. This limits the amount of STP that we have been able to expose to the number of dynamic instructions between the launch point and the original task call site, which we call the $L P$ overlap. The instructions represented by the task overlap, between the beginning of the speculative task and the earliest profiled conflict destination, are profiled memory-independent of all of the preceding code. By using explicit additional synchronization around the earliest profiled conflict destination, early start with synchronization enables the task overlap to contribute to the number of exposed memory-independent instructions.

\subsubsection{Early Start with Synchronization}

Currently, a task with a large task overlap and a small LP overlap would not be selected as memoryindependent, even though a large portion of the task is memory-independent with its preceding code. By synchronizing the control or memory dependence with the earliest conflict destination, the task may be launched earlier than the dependence. Where possible, we placed the task launch point above the dependence a distance equal to the task overlap. Any control dependences between the new task launch point and the synchronization point would be handled as speculative control dependences.

Figure 7 illustrates synchronization points. When the dependence determines a launch point, in Figure 7(b), all memory-independent instructions come from the LP overlap. Figure 7(c) shows that by synchronizing the dependence with the earliest conflict destination, both the LP overlap and the task overlap contribute to the number of memory-independent instructions.

Early start shows the greatest increase in parallelism so far, exposing on the average an additional $6.6 \%$ of dynamic instructions as memory-independent (the fifth bar per benchmark of Figure 6). The big increase in parallelism came from tasks that had not previously exhibited a significant level of STP, but now are able to exceed our thresholds.

The extra parallelism exposed through early start will come at the cost of additional dynamic instructions and the cost of explicit synchronization. We did not impose any penalties to simulate those costs as they will be architecture-dependent.

\subsection{Memory Granularity}

We define a memory access conflict to occur with two accesses to the same memory region. The memory granularity (the size of these regions) effects the amount of parallelism that is exposed. Reasonable gran- 


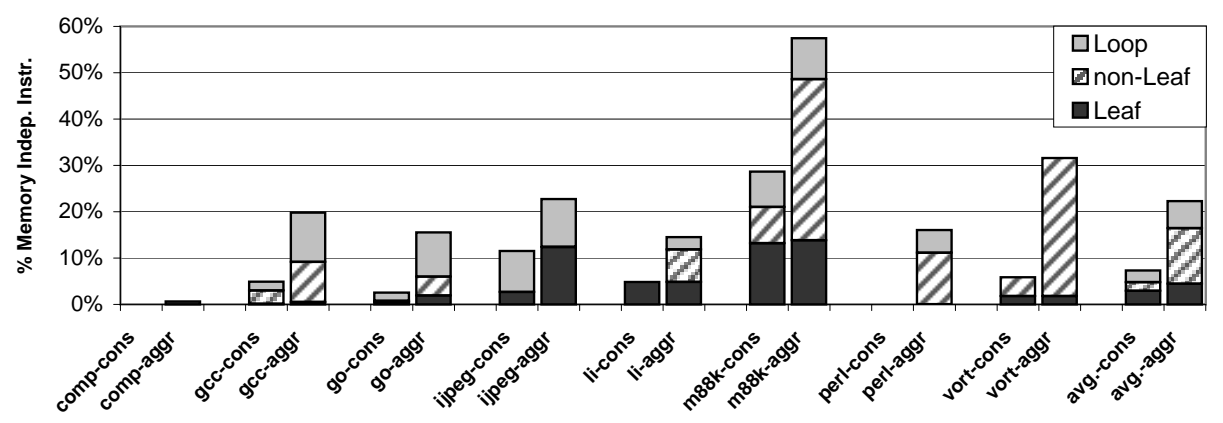

Figure 8: Conservative vs. Aggressive Speculation: Conservative is page-level profiling on all task types with static control dependence and profiled memory dependence. Aggressive is word-level profiling on all task types with profiled control dependence, speculative memory dependence, and early start.

ularities are full bytes, words, cache-lines, or pages. When a larger memory granularity is used, this may result in a conservative placement of launch points. The actual granularity used will depend on the granularity at which the processor can detect memory ordering violations. Managing profiled-parallel tasks whose launch points were determined with a memory granularity of a page would allow the use of existing page protection mechanisms to detect and recover from dependence violations. Thus, our starting configuration used page-level profiling. We also investigate word-level profiling.

In Figure 6, the last bar shows the results of word-level profiling on top of profiled control dependence, speculative memory dependence and early start. The average gain in memory-independence across all benchmarks was about $6 \%$ of dynamic instructions.

\subsection{Experiment Summary}

Figure 8 re-displays the extremes of our STP results from conservative to aggressive speculation broken down by task types. The conservative configuration includes page-level profiling on all task types with static control dependence and profiled memory dependence. The aggressive configuration comprises wordlevel profiling on all task types with profiled control dependence, speculative memory dependence, and early start. M88ksim showed the largest increase in the percentage of memory-independent instructions at over $28 \%$, with vortex very close at over $25 \%$, and the average across benchmarks at about $14 \%$. Each of these increases in parallelism were largely seen in the non-leaf procedures. Ijpeg was the only benchmark to see a sizable increase contributed by leaf procedures. Loops accounted for increases in gcc, go, li and perl.

Table 1 displays statistics from the conservative and aggressive speculation of those tasks which exceed our thresholds. The average overlap is that part of the average task length that can be overlapped with other execution. The number of tasks selected for STP is greatly affected by aggressive speculation.

In this Section, early start with synchronization provided the highest single increase among all alternatives. Speculative systems with fast synchronization should be able to exploit STP the most effectively. Our results also indicate that a low-overhead word-level scheme to exploit STP would be profitable.

\section{Implementation Issues}

For our limit study of Speculative Task Parallelism, we have assumed a hypothetical speculative machine that supports speculative futures with mechanisms for resolving incorrect speculation. When implementing this machine, a number of issues need to be addressed. 


\begin{tabular}{|c|c|c|c|c|c|c|}
\hline & \multicolumn{3}{|c|}{ Conservative } & \multicolumn{3}{|c|}{ Aggressive } \\
\hline & Selected & Avg Dynamic & Average & Selected & Avg Dynamic & Average \\
\hline & Tasks & Task Length & Overlap & Tasks & Task Length & Overlap \\
\hline compress & $\overline{0}$ & $\overline{0}$ & 0 & 1 & $\overline{282}$ & $\overline{42}$ \\
\hline $\mathrm{gcc}$ & 22 & 412 & $\overline{93}$ & 74 & 363 & 89 \\
\hline go & 12 & 546 & 118 & 49 & 334 & 76 \\
\hline ijpeg & 5 & 803 & 300 & 12 & 996 & 266 \\
\hline li & 3 & 50 & 50 & 9 & 14198 & 55 \\
\hline m88ksim & 5 & 34 & 29 & 20 & 123 & 43 \\
\hline perl & 0 & 0 & $\overline{0}$ & 10 & 515 & $\overline{42}$ \\
\hline vortex & 14 & 114 & 45 & $\overline{92}$ & 215 & 47 \\
\hline average & 8 & 245 & 79 & 33 & 2128 & 83 \\
\hline
\end{tabular}

Table 1: Task Statistics (Conservative vs. Aggressive)

\section{Speculative Thread Management}

Any system that exploits STP would need to include instructions for initialization, synchronization, communication and termination of threads. As launched tasks may be speculative, any implementation would need to handle mis-speculations.

Managing speculative tasks would include detecting load/store conflicts between the preceding code and the launched task, buffering stores in the launched task, and checking for memory-independence before committing the buffered stores to memory. One conflict detection model includes tracking the load and store addresses in both the preceding code and the launched task. The amount of memory-independence accommodated by this model will be determined by the size and access of load-store address storage, and the conflict granularity.

Another conflict detection model uses a system's page-fault mechanism. When static analysis can determine the page access pattern of the preceding code, the launched task is given restricted access to those pages, while the preceding code is given access to only those pages. Any page access violation would cause the speculative task to fail.

\section{Inter-thread Communication}

Any implementation that exploits STP will benefit from a system with fast communication between threads. At the minimum, inter-thread communication is needed at the end of a launched task and when the task results are used. Fast communication would be needed to enable early start with synchronization. The ability to quickly communicate a mis-speculation would reduce the number of instructions that are issued but never committed. This is especially important for systems where threads compete for the same resources.

\section{Adaptive STP}

We select tasks that exhibit STP based upon memory access profiling and compiler analysis. The memory access pattern from one dataset may or may not be a good predictor for another dataset. Two feedback opportunities arise that allow the execution of another data set to adapt to differences from the profiled dataset. The first would monitor the success rate of particular launch points, and suspend further launches when it fails too frequently.

The second feedback opportunity is found in continuous profiling. Rather than have a single dataset dictate the launched tasks for all subsequent runs, let datasets from all previous runs dictate the launched tasks for the current run. It is possible that the aggregate information from the preceding runs would have a better predictive relationship with future runs. Additionally, the profiled information from the current run 
could be used to supersede the profiled information from previous runs, with the idea that the current run may be its own best predictor. Although profiling is expensive and must be optimized, the exact cost is beyond the scope of this paper.

\section{Summary}

Traditional parallel compilers do not effectively parallelize irregular applications because they contain little loop-level parallelism due to ambiguous memory references. A different source of parallelism, namely Speculative Task Parallelism arises when a task (either a leaf-procedure, a non-leaf procedure or an entire loop) is control- and memory-independent of its preceding code, and thus could be executed in parallel. To exploit STP, we assume a speculative machine that supports speculative futures (a parallel programming construct that executes a task early on a different thread or processor) with mechanisms for resolving incorrect speculation when the task is not, after all, independent. This allows us to speculatively parallelize code when there is a high probability of independence, but no guarantee.

Through profiling and compiler analysis, we find memory-independent tasks that have no memory conflicts with their preceding code, and thus could be speculatively executed in parallel. We estimate the amount of STP in an irregular application by measuring the number of memory-independent instructions these tasks expose. We vary the level of control dependence and memory dependence to investigate their effect on the amount of memory-independence we found. We profile at different memory granularities and introduced synchronization to expose higher levels of memory-independence.

We find that no one task type exposes significantly more memory-independent instructions, which strongly suggests that all three task types should be profiled for STP. We also find that starting a task early with synchronization around dependences exposes the highest additional amount of memory-independent instructions, an average across the SPECint 95 benchmarks of $6.6 \%$ of profiled instructions. Profiling memory conflicts at the word-level shows a similar gain in comparison to page-level profiling. Speculating beyond profiled memory and static control dependences shows the lowest gain which is modest at best. Overall, we find that 7 to $22 \%$ of instructions are within memory-independent tasks. The lower amount reflects tasks launched in parallel from the least speculative locations.

\section{Acknowledgments}

This work has been supported in part by DARPA grant DABT63-97-C-0028, NSF grant CCR-980869, equipment grants from Compaq Computer Corporation, and La Sierra University.

\section{References}

[1] H. Akkary and M. Driscoll. A dynamic multithreading processor. In 31st International Symposium on Microarchitecture, Dec. 1998.

[2] R. Alverson, D. Callahan, D. Cummings, B. Koblenz, A. Porterfield, and B. Smith. The Tera computer system. 1990 International Conf. on Supercomputing, June 1990.

[3] M. Franklin and G. S. Sohi. ARB: A hardware mechanism for dynamic reordering of memory references. IEEE Transactions on Computers, May 1996.

[4] D. M. Gallagher, W. Y. Chen, S. A. Mahlke, J. C. Gyllenhaal, and W. W. Hwu. Dynamic memory disambiguation using the memory conflict buffer. In Proceedings of the 6th International Conference on Architecture Support for Programming Languages and Operating Systems, Oct. 1994.

[5] L. Gwennap. Intel discloses new IA-64 features. Microprocessor Report, Mar. 81999. 
[6] L. Hammond, M. Willey, and K. Olukotun. Data speculation support for a chip multiprocessor. ACM SIGPLAN Notices, 33(11):58-69, Nov. 1998.

[7] S. Keckler, W. Dally, D. Maskit, N. Carter, A. Chang, and W. Lee. Exploiting fine-grain thread level parallelism on the MIT Multi-ALU processor. In Proceedings of the 25th Annual International Symposium on Computer Architecture (ISCA-98), pages 306-317, June 1998.

[8] M. S. Lam and R. P. Wilson. Limits of control flow on parallelism. In Proceedings of the 19th Annual International Symposium on Computer Architecture (ISCA-92), May 1992.

[9] R. H. Litton, J. A. D. McWha, M. W. Pearson, and J. G. Cleary. Block based execution and task level parallelism. In Australasian Computer Architecture Conference, Feb. 1998.

[10] P. Marcuello and A. Gonzalez. Clustered speculative multithreaded processors. In Proceedings of the [ACM] International Conference on Supercomputing, June 1999.

[11] P. Marcuello and A. Gonzalez. Exploiting speculative thread-level parallelism on a SMT processor. In Proceedings of the International Conference on High Performance Computing and Networking, April 1999.

[12] A. Moshovos, S. E. Breach, T. N. Vijaykumar, and G. S. Sohi. Dynamic speculation and synchronization of data dependences. In Proceedings of the 24th Annual International Symposium on Computer Architecture (ISCA-97), June 1997.

[13] S. S. Muchnick. Advanced Compiler Design and Implementation. Morgan Kaufmann Publ., San Francisco, 1997.

[14] J. T. Oplinger, D. L. Heine, and M. S. Lam. In search of speculative thread-level parallelism. In Proceedings of the 1999 International Conference on Parallel Architectures and Compilation Techniques (PACT99), October 1999.

[15] J. T. Oplinger, D. L. Heine, S. Liao, B. A. Nayfeh, M. S. Lam, and K. Olukotun. Software and hardware for exploiting speculative parallelism with a multiprocessor. Technical Report CSL-TR-97-715, Stanford University, Computer Systems Laboratory, 1997.

[16] G. S. Sohi, S. E. Breach, and T. N. Vijaykumar. Multiscalar processors. In Proceedings of the 22nd Annual International Symposium on Computer Architecture (ISCA-95), June 1995.

[17] A. Srivastava and A. Eustace. ATOM: A system for building customized program analysis tools. Research Report 94.2, COMPAQ Western Research Laboratory, 1994.

[18] J. G. Steffan and T. C. Mowry. The potential of using thread-level data speculation to facilitate automatic parallelization. In Proceedings of the 4th International Symposium on High-Performance Computer Architecture, Feb. 1998.

[19] D. M. Tullsen, S. J. Eggers, and H. M. Levy. Simultaneous multithreading: Maximizing on-chip parallelism. In Proceedings of the 22nd Annual International Symposium on Computer Architecture (ISCA-95), June 1995.

[20] T. N. Vijaykumar and G. S. Sohi. Task selection for a multiscalar processor. In 31st International Symposium on Microarchitecture, Dec. 1998.

[21] S. Wallace, B. Calder, and D. Tullsen. Threaded multiple path execution. In Proceedings of the 25th Annual International Symposium on Computer Architecture (ISCA-98), June 1998. 